\title{
The Histological Origin of Solid-Pseudopapillary Neoplasm of the Pancreas
}

\author{
Zuo-Xing Niu, Chang-Liang Wang, Wen-Hua Zhao, M.M. Ai-juan Tian, Lan-ping Sun, Yu-hui Li, \\ Bo Zhang, Wei-xia Zhong and Sheng $\mathrm{Li}^{*}$
}

Shandong Tumor Hospital, Jinan 250117, Shandong Province, China

\begin{abstract}
Solid-pseudopapillary neoplasm of the pancreas (SPNP), as a distinct tumor, often occurred in young female patients with both exocrine and endocrine differentiation. Our aim was to define the SPNP histogenesis and investigate the relationships between its histological origin and the sexual hormone receptors. 20 tumor samples were subjected to the analysis of immunohistologic staining patterns and presence of sex hormone receptors and other markers of SPNP. Immunohistological results indicated that the tumors were positive for alpha-1-antitrypsin (17 cases), vimentin (14 cases), synaptophysin (10 cases), chromaffin granule A (5 cases), cytokeratin and insulin (2 cases), glucagon and S-100 (1 case), progesterone-receptor protein (14 cases), estrogen receptor protein (1 case), pS2 (6 cases), but negative for carcinoembryonic antigen, gastrin and androgen-receptor protein. SPNP is closely associated with the sex hormone receptor.
\end{abstract}

Keywords: Pancreatic neoplasm, solid-pseudopapillary neoplasms, immunohistochemistry, sex hormone receptors, molecular markers.

\section{INTRODUCTION}

Solid-pseudopapillary neoplasm of the pancreas (SPNP) is a rare tumor of unidentified histological origin. Since the first description of the neoplasm by Frantz in 1959 [1]. SPNP has often been misdiagnosed as adenocarcinoma, cystadenomas, papillary cystadenocarcinoma, or cystadenocarcinoma. The clinical picture and pathological features of SPNP can be mistaken for other pancreatic tumors, such as pancreatic cancer, non-functioning islet cell tumor, etc, although it usually follows a benign clinical course, In 2000, WHO renamed the disease, solidpseudopapillary neoplasm of the pancreas [2]. Because SPNP occurs predominantly in young women, genetic and/or gender-specific factors may play a role in the disease pathogenesis. However, there is a lack of direct evidence to support such a conclusion and more efforts are needed to elucidate the pathogenesis of SPNP. Using histological and immunohistochemical methods, we analyzed the clinical data from 20 cases (female 18, male 2) with SPNP to investigate the SPNP histogenesis and relationship between immunohistologic staining patterns and the presence of sex hormone receptors in SPNP.

\section{PATIENTS AND METHODS}

Between January 1995 and December 2003, twenty patients (female 18, male 2) with a diagnosis of SPNP who were treated at the Shandong Tumor Hospital (8 cases), People's Hospital of Heze City (4 cases), Qilu Hospital (3 cases), Tumor Hospital of Dezhou City (2 cases), Central Hospital of Feicheng Mining Group Co., Ltd (2 cases) and the People's Hospital of Linxi City (1 case) were enrolled in the study. We reviewed 689 patients with peripancreatic malignancies who were admitted to the above-mentioned

\footnotetext{
*Address correspondence to this author at the Shandong Tumor Hospital, Jinan 250117, Shandong Province, China; Tel: +86-13705311949; Fax: +86-531-87984079; E-mail: drlisheng@sohu.com
}

hospitals, however, only 20 were diagnosed with SPNP (2.9\%). Their clinically pathological data and follow-up information were available. Three pathologists reexamined the pathological specimens of the patients using light microscope and made diagnosis according to WHO Tumor Classification (2000). The neoplasms consisted of 3 distinct histological components: papillary, cystic, and solid. In the papillary areas, neoplastic cells formed characteristic papillary structures around a central fibrovascular stalk. The areas of cystic degeneration stained with alcian blue showed the presence of giant cells, and cholesterin crystals. The solid areas had a unique pseudorosette pattern, which is similar to that of islet cell neoplasms.

Immunohistological analyses were performed using a streptavidin peroxidase conjugated method (SP method). Antibodies against the following proteins were used: alpha1-antitrypsin ( $\alpha-1-\mathrm{AT})$, cytokeratin (CK), synaptophysin, S100 protein, $\mathrm{CgA}$, insulin, glucagons, gastrin, vimentin, carcino-embryonic antigen (CEA), estrogen receptor (ER), progesterone receptor (PR), androgen-receptor (AR) and $\mathrm{pS} 2$ proteins (Maixin Biological Technology Co., Ltd. Fuzhou). Pancreatic tissue was used as a positive control for $\alpha-1-A T$, CK, S-100 protein, chromaffin granule, insulin, glucagons, gastrin, vimentin, CEA, and synaptophysin. Prostate tissue was used as a positive control for AR protein; breast tissue was used as a positive control for ER, PR and PS2 proteins. Normal mouse immunoglobulin $G$ was substituted for the primary antibody as the negative control. Subsequently, immunohistochemical staining patterns of the neoplasms obtained from the 2 male and 18 female patients were compared.

\section{RESULTS}

\section{Clinicopathological Characteristics}

Clinical information on the 20 patients with SPNP was available. The patients included 2 Chinese men and 18 Chinese women (age range 13-48 years with a median age of 
25.3 years). The disease onset age of these patients ranged between 13 and 28 years of age, which accounted for $70 \%$ $(14 / 20)$. The median size of the lesions was about $8.2 \mathrm{~cm}$ (range, 3-17 cm) in diameter. The first symptoms of the patients comprised abdominal masses (10 cases) and abdominal pain ( 9 cases). 3 cases were characterized by associated nausea and occasional vomiting, as well as discomfort of the epigastric region (1 case). There was no history of diabetes or pancreatic insufficiency. All cases were examined by ultrasonic B and CT. 12 cases showed mixed cystic and solid masses, 7 cases showed solid mass and 1 case showed cystic mass.

5 patients had their primary neoplasm within the head, 2 in the body and 5 in the body and tail of pancreas, 6 in the tail of the gland, 1 in the mesentery and 1 behind the abdominal membrane. There were 9 cases with lesions adherent to surrounding tissues and organs, 1 case of lymphadenectasis occurred around the pancreas, 4 cases had metastasis to portal vein, 1 case to the gastric wall, 2 cases to the duodenum, 1 case to the spleen and 1 case extensively spreaded into the abdominopelvic cavity. 9 cases were cured by simple excision, 4 cases by duodenopancreatectomy (PPPD), and 4 cases by excision of spleen and distal pancreatectomy, 3 cases by alleviative tumor excision. Thereafter, 2 cases received adjunctive therapy after the operations. 1 case was subjected to $\gamma$ ray radiation because the surrounding tissues of the lesion were closely adjacent to the superior mesenteric artery and the tumor therefore could not be cut. After radiation therapy, the masses contracted. Another case was cured after transcatheter hepatic arterial chemoembolization because of liver metastasis and the symptoms were alleviated. (The clinical data of 20 cases with SPNP was shown in Table 1)

Table 1. Clinical Data of 20 Cases with SPNP

\begin{tabular}{|lc|}
\hline Gender & Case \\
\hline Female & $18(90 \%)$ \\
Male & $2(10 \%)$ \\
\hline Age (mean) & \\
13-48 years & (mean,25.3 years) \\
\hline Tumor Size & \\
3-17 cm & 1 \\
\hline Gross Apearance & \\
Cystic & 7 \\
Solid & 12 \\
Cystic and Solid & \\
\hline Surgical intervention & 9 \\
Simple excision & 4 \\
Duodenopancreatectomy & 4 \\
Excision of spleen and distal pancreatectomy & 3 \\
Alleviative tumor excision & \\
\hline
\end{tabular}

17 cases were given follow up and 14 of the cases are still living. The follow up period ranged from 9-120 months and the average surviving period was 44.4 months. There were 10 cases living without tumor reoccurrence; 1 case had liver metastasis 46 months after tumor excision and the patient still lived (more than 23 months) with the tumor; the disease relapsed in 1 case 24 months after the operation, and the patient lived for 21 months after the second operation for tumor excision; 2 cases ( 1 case of them accompanied liver and pelvic cavity metastases) were cured by cytoreductive surgery, and they were still living with the tumors until the follow-up day for 9 and 30 months, respectively. 3 cases died 12, 20 and 25 months later after the tumor excision due to tumor reoccurrence, respectively.

\section{Pathological Examinations}

Tumors of the patients were generally large in size; color varied from gray yellow, gray red to dark red, and showed irregular cystic cavities lined by soft, friable tissue, accompanied by bleeding and necrosis. Old blood-like liquid was observed in the cystic part and the contents were similar to fluffed cotton or sponge. The thickness of the capsule wall was $0.2-1.8 \mathrm{~cm}$ and the inner walls were coarse 12 cases had cystic and solid tumors, 7 cases had solid tumors, and 1 case showed a pure cystic tumor. 17 cases had tumors with fibrous membrane and distinct boundaries, in which there were tumors with complete capsules ( 9 cases) and tumors with partial capsules ( 8 cases). In 3 cases, tumors had no capsule and encroached the surrounding tissues.

The microscopic examination of the tumors confirmed the characteristic microscopic features of SPNP. The solid regions were composed of tumor cells with relatively consistent sizes and shapes. The tumor cells were medium sized, polygon shaped or stretched longer, and the cytoplasm was a light eosinic color. In addition, some parts of the cytoplasm were transparent, and the cell boundary was clear. The nucleus was round or ovate and the nuclear chromatin was fine and evenly distributed. Small nucleoli could be observed in 12 cases $(60 \%)$ and nuclear grooves could be found in 16 cases $(80 \%)$ (Figs. 1, 2). Caryocinesis could be observed occasionally, among which 5 cases $(25 \%) \leq 1$ /10HPF, 2 cases $(10 \%) \leq 3 / 10 \mathrm{HPF}$. Many supporting vessels were far from the swelling cells and degenerated small vessels were observed in the tumor parenchyma. The juxtavascular cells were well maintained and the tumor cells surrounding the stratified layer of the fibrous vessels formed characteristic pseudo-mammillae (Fig. 3). The interstitial substance surrounding the small vessels appeared to be mucinous degeneration. Some tumor cells formed pseudochrysanthemum groups surrounding the vessels (Fig. 4) and some eosinophilic bodies could be observed between the cells, or some cells near the end of the vessel were radiatively arranged in regions far from vessels. There were 14 cases $(70 \%)$ with tumor bleeding, necrosis and 14 cases $(70 \%)$ with mucinous degeneration of interstitial substances. There were still 6 cases $(30 \%)$ with foam cells, cholesterol crystallization and foreign-body giant cell reaction and 2 cases $(10 \%)$ with calcification. Although there was apparent gross circumscription, the microscopic interface between the tumors and the adjacent pancreas commonly showed an infiltrative growth pattern with islands of non-neoplastic pancreatic parenchyma entrapped within the tumor and nests of tumor cells extending into the adjacent pancreas. 11 cases $(55 \%)$ had infiltrated pancreatic parenchyma, among which 7 cases $(35 \%)$ represented metastasis to nerves and 2 cases 
(10\%) represented metastasis to the duodenum. Tumor metastasis occurred in the liver, peripanchreatic lymph nodes, and spleen parenchyma in one case each.

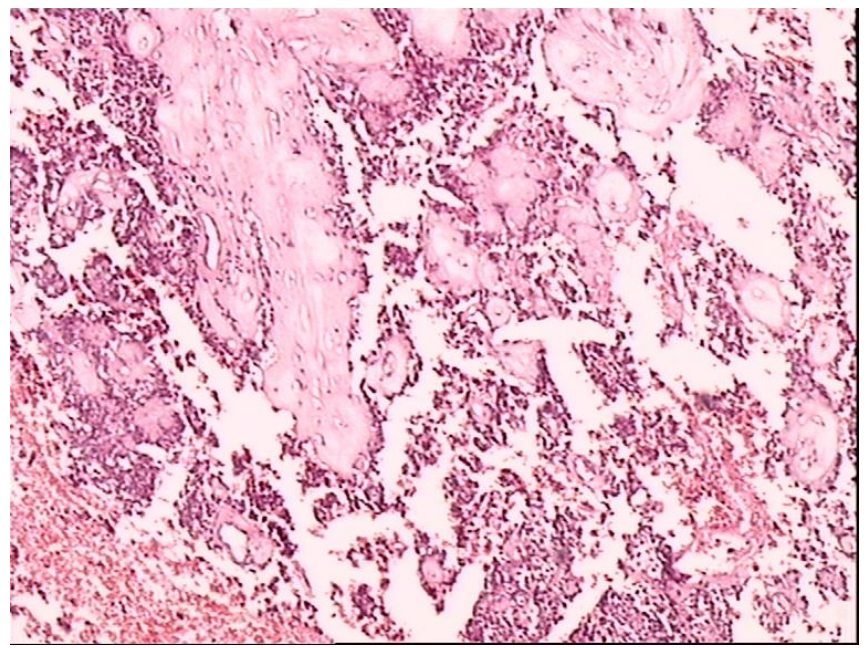

Fig. (1). The tumor cells formed thick pseudo- papillae. The center of was characterized by fibrosis and hyalinizination. Among the papillae, the gaps that formed after cell degeneration were observed. HEx40.

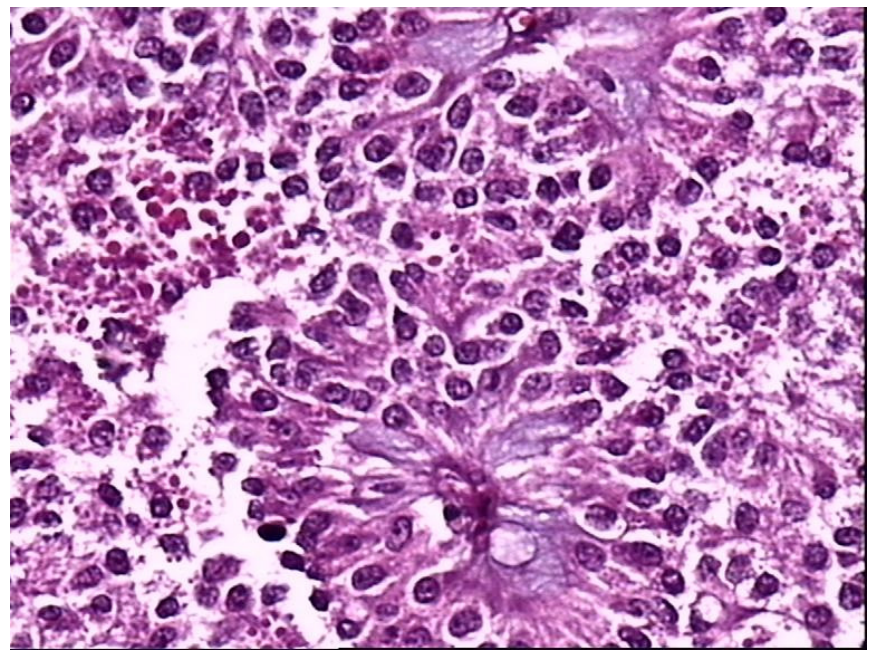

Fig. (2). The tumor cells were relatively medium sized and shaped, and the cell boundary was clear. The nucleus was round or ovate and the nuclear chromatin was fine and evenly distributed. Small nucleoli, nuclear grooves and eosinophilic bodies could be observed.

Tissue sections from all 20 patients were subjected to immunohistologic staining. None of the neoplastic cells was stained with periodic acid-Schiff, alcian blue ( $\mathrm{pH} \mathrm{2.5).} 17$ cases showed $\alpha$-1-antitrypsin positive staining $(85 \%)$ and 14 cases showed vimentin staining $(70 \%)$ (Figs. 5, 6), 10 cases $(50 \%)$ showed synaptophysin positive staining (Fig. 7), 5 cases $(25 \%)$ had focal chromaffin granule weak positive, 2 cases $(10 \%)$ showed cytokeratin positive staining, 2 cases were positive for insulin $(10 \%)$ and 1 case was positive for glucagon $(5 \%)$. 1 case $(5 \%)$ showed S-100 protein positive expression, and14 cases (70\%) showed extensive PR expression (Fig. 8). 1 case (5\%) and 6 cases (30\%) showed of ER and pS2 protein positive staining, respectively. None of the patients showed androgen-receptor protein, carcinoembryonic antigen and gastrin positive staining (Table 2).

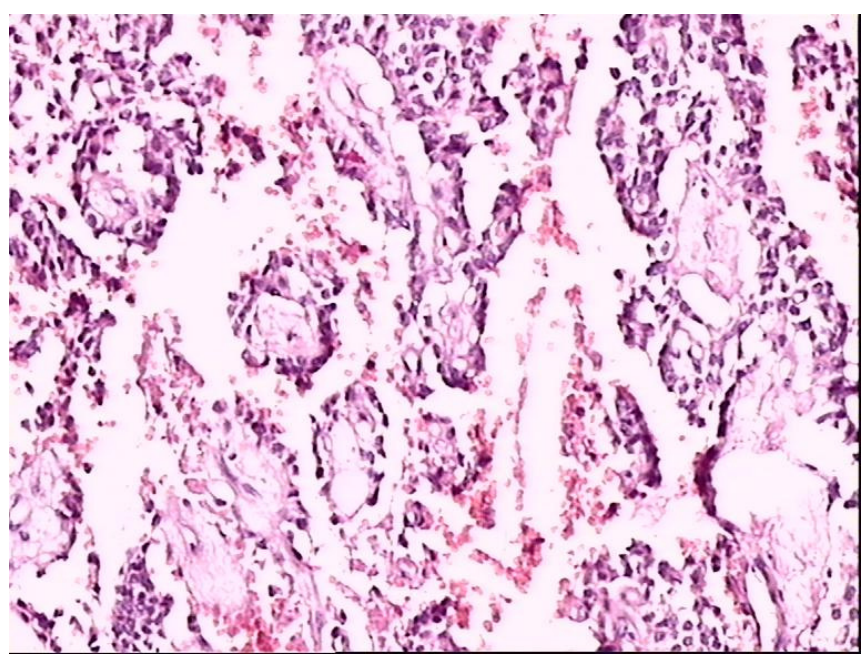

Fig. (3). The tumor cells surrounding the stratified layer of the fibrous vessels formed characteristic pseudo-mammillae, HEx100.

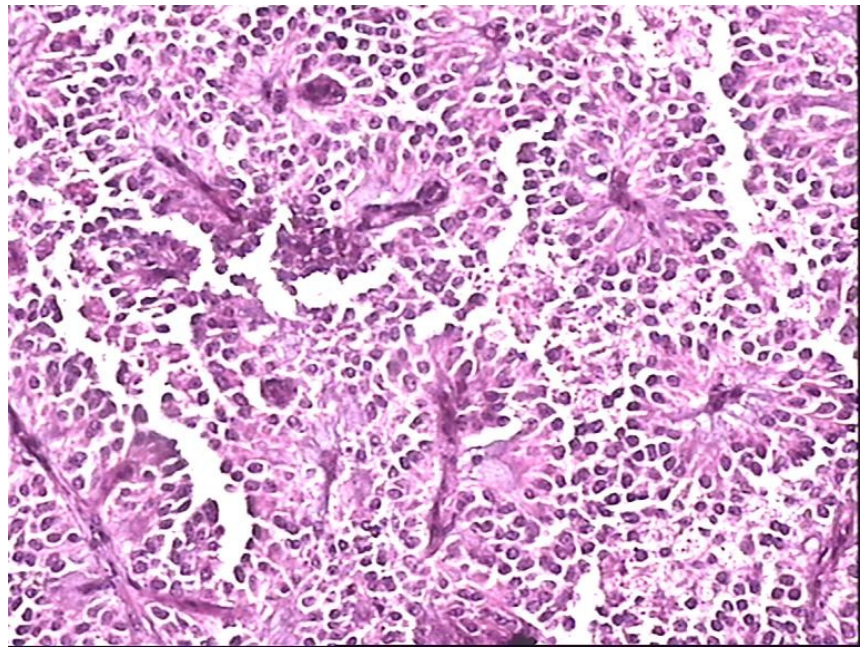

Fig. (4). The tumor cells formed pseudo-chrysanthemum group surrounding the vessels. HEx 400.

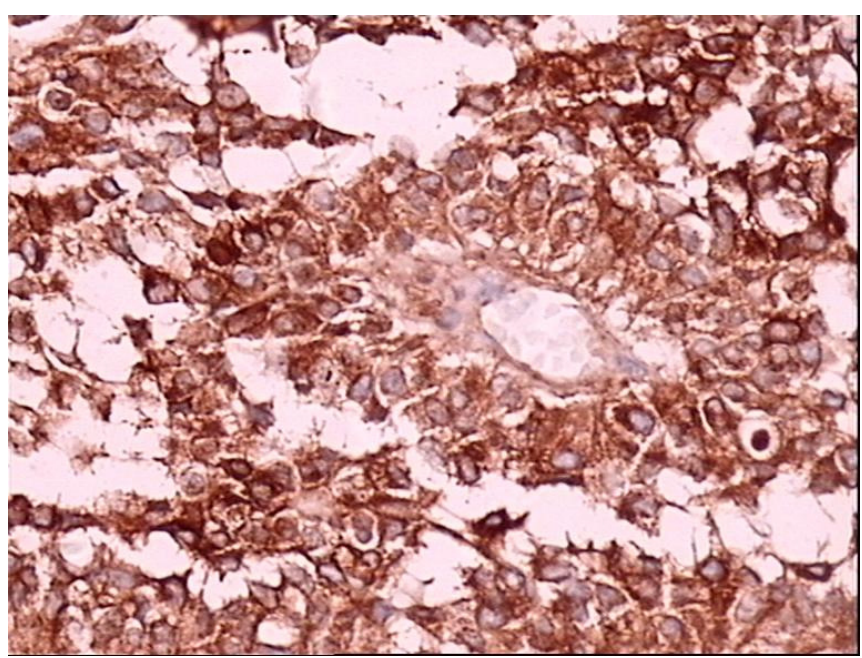

Fig. (5). The $\alpha$-1-antitrypsin of tumor cells was extensively positive. SP x 400. 


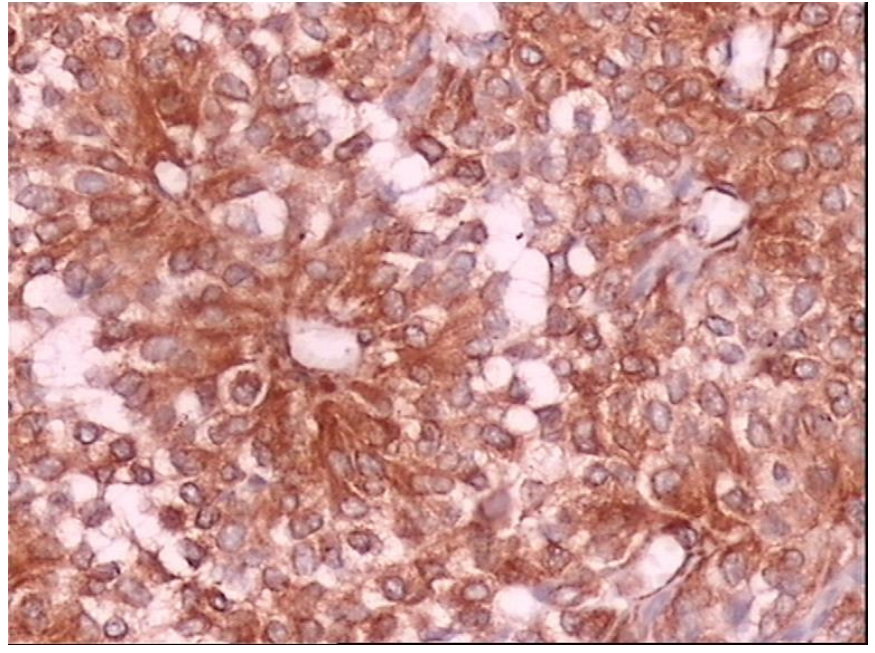

Fig. (6). The vimentin of tumor cells was extensively positive. SP $\mathrm{x}$ 400 .

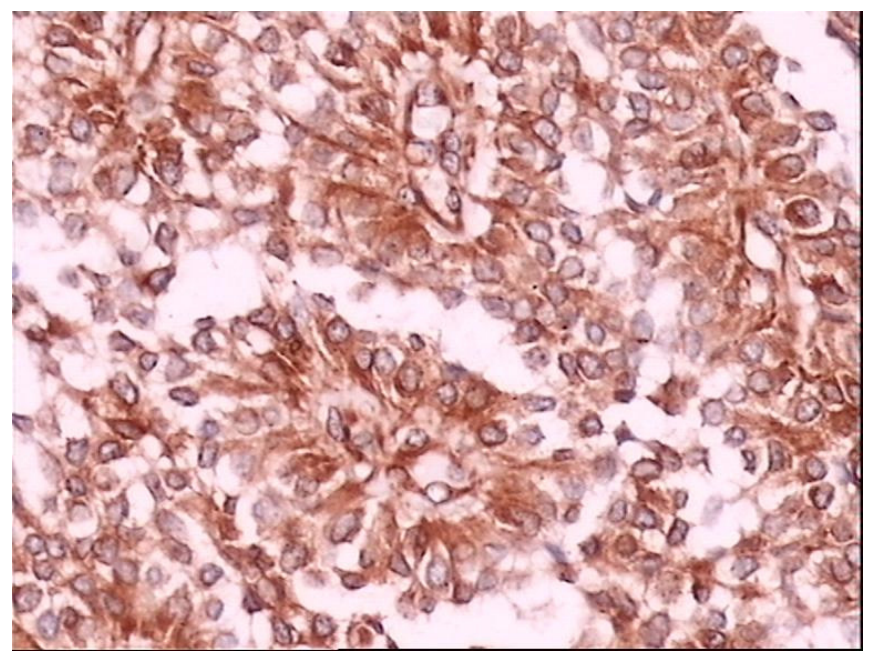

Fig. (7). The synaptophysin of most tumor cells were positive, SP x 400 .

\section{DISCUSSION}

Most of the patients in this series presented with abdominal masses or vague mild abdominal pain. Their clinical symptoms were often vague and nonspecific, leading to a delay in diagnosis. As a result, the tumor was quite large in size, as observed in our experience. Size of the lesion is not a predictor of unresectability. Lesions ranging from 10 to $30 \mathrm{~cm}$ in size can still be resected. Tumors can occur in any part of the pancreas. Most cases in our study showed that tumor sites located in the tail of the pancreas, and the secondary common region was the head of pancreas $[3,4]$.

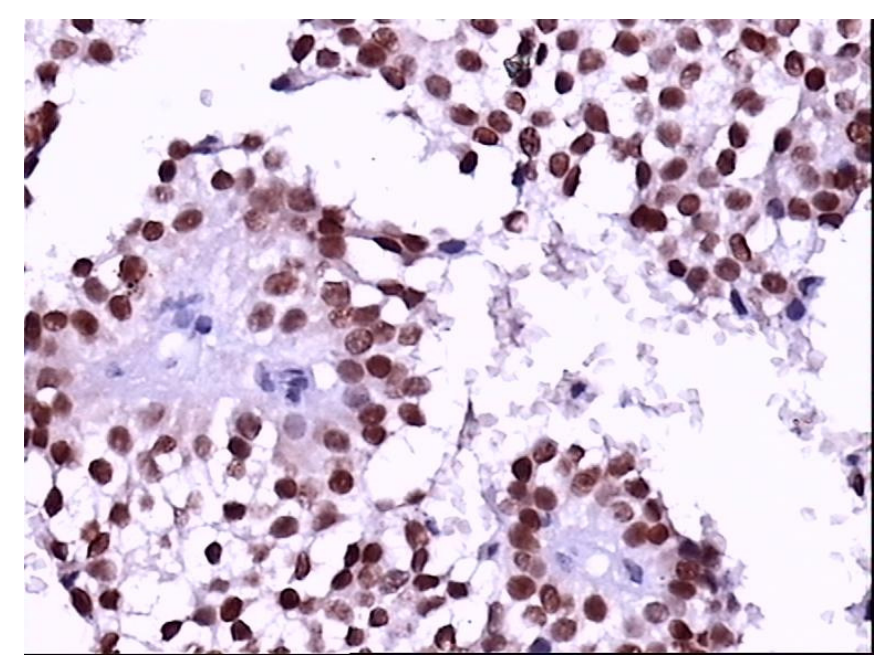

Fig. (8). The tumor cells showed extensively strong PR positive staining. SP x 200.

Diagnosis is often made incidentally at abdominal examination, during imaging studies or laparatomy conducted to exclude other pathology. Less SPNP can be detected during an evaluation for trauma after the occurrence of a tumor-related complication, such as intratumoural or intraperitoneal hemorrhage, or during a gynecological exam. Although the diagnosis is rarely made pre-operatively, characteristic computed axial tomography and magnetic resonance imaging scans combined with age and sex profile should be sufficient for the decision to operate. Patients with malignant SPT should have careful follow-up [5-7]. No abnormalities in laboratory tests, such as pancreatic enzymes, tumor markers for pancreatic cancer (CA19-9, alpha-fetoprotein, carcinoembryonic antigen) or endocrine tests, are easily observed.

In our study, the pathologic features of SPNP mainly consist of cystic and solid tumors. Microscopic analysis indicated that the cytoplasm was a light eosin color or transparent and the nucleus was round or ovate. Nuclear groove and small nucleolus could be found. Tumor cells surrounding the stratified layer of the fibrous vessels formed pseudo-mammillae, a characteristic change of SPNP.

Some studies suggest there are some methods to distinguish SPNP from neuroendocrine tumor $[6,8]$. In our study, $\alpha$-1-antitrypsin was positive in the acinic cell carcinoma. The disease could express regional or general positive NSE [9]. It was reported that the positive rate of vimentin was relatively high in SPNP patients $[9,10]$. Very few cases expressed positive S-100 protein $[10,11]$. CEA and CA19-9were high sensitive markers of the pancreatic duct adenoma and the acinous cells and endocrine cells were

Table 2. The Immunohistochemical Staining of the 20 Patients (Female 18, Male 2) with SPNP

\begin{tabular}{|c|c|c|c|c|c|c|c|c|c|c|c|c|c|c|}
\hline \multirow[b]{2}{*}{ Antigen } & \multicolumn{14}{|c|}{ Immunohistochemical Staining } \\
\hline & a1-AT & Vit & SYN & $\mathrm{CgA}$ & $\mathrm{CK}$ & INS & GL & S-100 & PR & ER & $\mathrm{pS} 2$ & CEA & GA & $\mathrm{AR}$ \\
\hline Case number & 17 & 14 & 10 & 5 & 2 & 2 & 1 & 1 & 14 & 1 & 6 & 0 & 0 & 0 \\
\hline Staining results & +++ & +++ & ++ & + & ++ & +++ & +++ & ++ & +++ & ++ & ++ & - & - & - \\
\hline
\end{tabular}

Note: a1-AT, $\alpha 1$-antitrypsin; Vit, vimentin; SYN, synaptophysin; CgA, chromaffingranule; CK, cytokeratin; INS, insulin; GL, glucagon; S-100, S-100 protein; PR, progesterone receptor; ER, estrogen receptor; pS2, pS2 protein; CEA, carcinoembryonic antigen; GA, gastrin; AR, gastrin and androgen-receptor protein. - negative; + minimal staining; ++ moderate staining; +++heavy staining. 
absent in SPNP. Most studies found that expression of cytokeratins in SPNP is negative [10]. In our case group, the tumor cell expression of these proteins was primarily focally distributed with weak positive expression, and CEA expression could not be detected. There were 2 cases with strong insulin and 1 case with strong glucagon positive expression in the focus forms. They were localized in the tumor parenchyma rather than the tumor margins. The result demonstrated that a few SPNP might have distinct differentiation characteristics. Our immunohistochemical analysis supported that SPNP mainly expressed exocrine character and were accompanied by neuroendocrine differentiation. Therefore, we could consider their origin to be from pluripotent embryonic cells of the pancreas with multipotential differentiation. The cells of SPNP may originate from the ductular-centroacinar cell compartment. However, on the other hand, there is no evidence of clear-cut terminal differentiation to either acinar or endocrine cells. The cytological features and the low proliferation rates and malignancies are also not consistent with a stem cell origin. In addition, the strong sex-linked occurrence also supports a stem cell origin.

Hormone receptors have been extensively researched, but the results are still inconclusive. Most investigations have not demonstrated estrogen receptors (ER) in these tumors, while the presence of progesterone receptors (PR) have been found in numerous studies $[9,12-14,15]$. This observation is consistent with the clinical finding that SPNP is mainly found in adolescent girls and young women. In our reserch, the results suggested that SPNP was a sexual hormone dependent tumor in females; however, 2 male cases in our group were ER and PR negative. Further immunohistological examination of all 20 neoplasms showed polymorphic differentiation. However, there were no significant differences in immunohistological staining patterns between male- and female-derived neoplasms.

In most clinical reports on SPNP, no pathologic factors predictive of prognosis have been identified, but tumors of some cases could regionally relapse, metastasize, and death has been occasionally reported [16-18]. After the tumor was completely removed, $95 \%$ of the patients with SPNP were cured [19]. About 5\% of the tumor recurred regionally [20,21], $16 \%$ infiltrated the stomach, duodenum or main vessels, less than $7 \%$ that with liver metastasis [18, 22, 23]. Even if SPNP could invade other regions and relapse or metastasize regionally, the surviving period of the patient was still relatively long after the second operation. Therefore, SPNP was a tumor with with low malignant potential.

Adjuvant therapy has been used only in a small number of patients. Radiotherapy or hepatic artery embolism has been effective to some extent. In this group, 2 cases were subjected to adjunctive therapy after the operations, 1 case adopted $\gamma$-ray radiotherapy for the tumor could not be excised because the tumor was extensivlely adherent to the tissues closely associated with the mesenteric artery. All masses contracted. In one case, we treated with transcatheter hepatic arterial chemoembolization because of the liver metastasis after the operation, and the body condition of the patient was stable. Generally, the prognosis of most patients is excellent.

\section{ACKNOWLEDGEMENTS}

This work was supported by grants from the National Natural Science Foundation of China, (No.30571712) \& Department of Science \& Technology of Shandong Province No. (2005BS03003).

\section{REFERENCES}

[1] Lam KY, Lo CY, Fan ST. Pancreatic solid-cystic-papillary tumor: clinic pathologic features in eight patients from Hong Kong and review of the literature. World J Surg 1999; 23: 1045-50.

[2] Kloppel G, Luttges J, Klimstra D, et al. Solid-pseudopapillary neoplasm. In: Hamilton SR, Aaltonen LA, Eds. Would health organization classification of tumor. Pathology and genetics of tumors of the digestive system. ARC Press:Lyon 2000; pp. 246-8.

[3] Lee SE, Jang JY, Hwang DW, et al. Clinical features and outcome of solid pseudopapillary neoplasm: differences between adults and children. Arch Surg 2008; 143: 1218-21.

[4] Karagülle E, Yildirim E, Türk E, et al. Solid pseudopapillary tumor of the pancreas: a case report. Turk J Gastroenterol 2006; 17: 3169.

[5] Yang F, Jin C, Long J, et al. Solid pseudopapillary tumor of the pancreas: a case series of 26 consecutive patients. Am J Surg 2009 [Epub ahead of print].

[6] Lee SS, Byun JH, Park BJ, et al. Quantitative analysis of diffusionweighted magnetic resonance imaging of the pancreas: usefulness in characterizing solid pancreatic masses. J Magn Reson Imaging 2008; 28: 928-36.

[7] Lee JH, Yu JS, Kim H, et al. Solid pseudopapillary carcinoma of the pancreas: differentiation from benign solid pseudopapillary tumour using CT and MRI. Clin Radiol 2008; 63: 1006-14.

[8] Geers C, Moulin P, Gigot JF, et al. Solid and pseudopapillary tumor of the pancreas--review and new insights into pathogenesis. Am J Surg Pathol 2006; 30: 1243-9.

[9] Aydiner F, Erinanç H, Savaş B, et al. Solid pseudopapillary tumor of the pancreas: emphasis on differential diagnosis from aggressive tumors of the pancreas. Turk J Gastroenterol 2006; 17: 219-22.

[10] Liu X, Rauch TM, Siegal GP, et al. Solid-pseudopapillary neoplasm of the pancreas: three cases with a literature review. Appl Immunohistochem Mol Morphol 2006; 14: 445-53.

[11] Dubova EA, Shchegolev AI, Mishnev OD, et al. Solid pseudopapillary tumor of the pancreas. Arkh Patol 2008; 70: 49-52.

[12] de Castro SM, Singhal D, Aronson DC, et al. Management of solid-pseudopapillary neoplasms of the pancreas: a comparison with standard pancreatic neoplasms. World J Surg 2007; 31: 11305 .

[13] Hao CY, Lu AP, Xing BC, et al. Solid pseudopapillary tumor of the pancreas: report of 8 cases in a single institution and review of the Chinese literature. Pancreatology 2006; 6: 291-6.

[14] Wang DB, Wang QB, Chai WM, et al. Imaging features of solid pseudopapillary tumor of the pancreas on multi-detector row computed tomography. World J Gastroenterol 2009; 15: 829-35.

[15] Santini D, Poli F, Lega S. Solid-papillary tumors of the pancreas histopathology. JOP 2006; 7: 131-6.

[16] Chen X, Zhou GW, Zhou HJ, et al. Diagnosis and treatment of solid-pseudopapillary tumors of the pancreas. Hepatobiliary Pancreat Dis Int 2005; 4: 456-9.

[17] Choi SH, Kim SM, Oh JT, et al. Solid pseudopapillary tumor of the pancreas: a multicenter study of 23 pediatric cases. J Pediatr Surg 2006; 41: 1992-5.

[18] Kim DH, Cho JH, Lee SH, et al. Two cases of pancreatic ductal adenocarcinoma, manifested as solid pseudopapillary tumor and intraductal papillary mucinous neoplasm. Korean J Gastroenterol 2008; 51: 142-6. 
[19] Kloppel G, Luttges J, Klimstra D, et al. Solid-pseudopapillary neoplasm. In: Hamilton SR, Aaltonen LA, Eds. Would health organization classification of tumor. Pathology and genetics of tumors of the digestive system. ARC Press: Lyon 2000; pp. 246-8.

[20] Madan AK, Weldo CB, Long WP, et al. Solid and papillary epithelial neoplasm of the pancreas. J Surg Oncol 2004; 85: 193-8.

[21] Murao T, Toda K, Tomiyama Y. Papillary and solid neoplasm of pancreas in a child: report of a case in which acinar differentiation was demonstrated by immunohistochemistry and elecron microscopy. Acta Pathol Jpn 1983; 33: 565-55.

[22] Sumida W, Kaneko K, Tainaka T, et al. Liver transplantation for multiple liver metastases from solid pseudopapillary tumor of the pancreas. J Pediatr Surg 2007; 42: 27-31.

[23] Yang F, Jin C, Long J, et al. Solid pseudopapillary tumor of the pancreas: a case series of 26 consecutive patients. Am J Surg 2009 [Epub ahead of print].

(C) Niu et al.; Licensee Bentham Open.

This is an open access article licensed under the terms of the Creative Commons Attribution Non-Commercial License (http://creativecommons.org/licenses/by$\mathrm{nc} / 3.0 /$ ) which permits unrestricted, non-commercial use, distribution and reproduction in any medium, provided the work is properly cited. 